Sharif University of Technology
Scientia Iranica
Transactions E: Industrial Engineering
wCIENTIA

\title{
Integrated forward-reverse logistics network design under uncertainty and reliability consideration
}

\author{
S.M. Hatefi ${ }^{\mathrm{a}, *}$, F. Jolai ${ }^{\mathrm{b}}$, S.A. Torabi ${ }^{\mathrm{b}}$ and R. Tavakkoli-Moghaddam ${ }^{\mathrm{b}}$ \\ a. Faculty of Engineering, Shahrekord University, Rahbar Boulevard, Shahrekord, P.O. Box 115, Iran. \\ b. School of Industrial Engineering, College of Engineering, University of Tehran, Tehran, Iran. \\ Received 9 September 2013; received in revised form 11 October 2014; accepted 7 February 2015
}

\section{KEYWORDS}

Integrated forwardreverse logistics network design; Network reliability; Facility disruptions; Robust optimization.

\begin{abstract}
This paper proposes a robust optimization model for robust and reliable design of an integrated forward-reverse logistics network with hybrid facilities under uncertainty and random facility disruptions. The proposed model utilizes several effective reliability strategies to mitigate the impact of random facility disruptions. First, the proposed model allows two types of hybrid facilities, namely, reliable and unreliable, to be located in the concerned logistics network where unreliable ones may be partially or fully disrupted, and thus a percentage of their capacities may be lost. However, they can still serve their customers with the remaining of their available capacities. Furthermore, a sharing strategy is taken into account, in which goods can be shipped from reliable hybrid facilities to unreliable ones to compensate their lost capacity. A robust optimization approach is applied on the developed model to handle the uncertainties in the parameters of the concerned network. Finally, several numerical experiments along with a sensitivity analysis are conducted to illustrate the significance and applicability of the proposed model as well as the effectiveness of the robust optimization approach in this context.

(C) 2016 Sharif University of Technology. All rights reserved.
\end{abstract}

\section{Introduction}

Recent studies in the supply chain literature pay a particular attention to designing integrated forwardreverse logistics networks to avoid sub-optimality resulting from the separated design of forward and reverse networks and, at the same time, reaching higher levels of productivity and customer satisfaction $[1,2]$. It has been recognized that robust and reliable design of such networks helps firms to maintain and enhance their competitive advantages, and assists them to cope with the growing environmental turbulence. A part of literature refers to configuration of integrated forward-reverse logistics networks, includ-

\footnotetext{
* Corresponding author. Tel./Fax: +983834424401 E-mail addresses: smhatefi@gmail.com (S.M. Hatefi); fjolai@ut.ac.ir (F. Jolai), satorabi@ut.ac.ir (S.A. Torabi); tavakoli@ut.ac.ir (R. Tavakkoli-Moghaddam)
}

ing both forward and reverse flows, because of the existing legal requirements, environmental protection, as well as related economic benefits $[3,4]$. The goal of a forward network is to provide a value for the end consumer in terms of product attributes like quality and cost, while the reverse network tries to recover the economic and environmental value from used products in a cost-effective manner. The processes and activities concerned with material supply, production, distribution, and consumption are embedded in the forward network, while reverse network encompasses the activities associated with collection, inspection/separation, recovery, and disposal of the used products $[5,6]$.

Another attention of the recent research is paid to incorporate risk management into the design phase of global supply chains. There are two wide categories of risks that impress supply chain network design problem: 
1. The risks originating from the difficulties in coordinating supply and demand;

2. The risks originating from the threat of disruptions to normal activities, which include the issues concerned with natural disasters, strikes, and economic disruptions, terrorist attacks, etc. [7].

Consequently, designing reliable and robust networks is of particular interest. It is worth pointing out the difference between robustness and reliability terms when studying supply chain risks. A supply chain is robust if it performs well when facing the first category of risks; while it is reliable if it performs well when disruptions occur, for example, when parts of the supply chain system fail due to natural disasters [8].

A large body of the extant literature is assigned to the first category of risks in which some parameters of a logistics network such as demand, lead times, transportation costs, and quantity of returned products may be uncertain. Environmental and system uncertainties are two aspects of uncertainty, which drastically affect the overall performance of supply chains [9]. The environmental uncertainty addresses uncertainties concerned with demand and supply originated in the performance of suppliers/manufacturers and behavior of customers. Those uncertainties impressing production, distribution, collection, and recovery processes are called system uncertainties. Since the logistics network design problem has a strategic nature, it is very critical that uncertainties are to be incorporated in the problem [9].

Stochastic programming, robust optimization, and fuzzy mathematical programming are three powerful tools, which can tackle the existing uncertainties in the parameters of the model. Application of stochastic programming for modeling uncertain supply chain network design can be seen in [4,10-15]. However, in many real applications of stochastic programming, there is no enough historical data to estimate the probability distribution of uncertain parameters. As an alternative, robust optimization can be applied to handle uncertainty in a given bounded uncertainty set. Implementation of robust optimization approach to design closed-loop logistics networks can be traced in $[16,17]$. In this paper, we resort to the robust optimization approach to handle uncertainties in the parameters of an integrated forward-reverse logistics network design problem. Furthermore, fuzzy mathematical programming is a flexible tool for modeling epistemic uncertainty that comes from lack of information about the actual value of parameters [18-20]. For effective implementation of fuzzy mathematical programing to design supply chain networks with uncertain parameters, the reader may refer to [21-23].

The second type of risks (our concern in this paper) can disrupt any point of a supply chain net- work (e.g., facilities and/or transportation links) in a relatively random manner. As highlighted by Peng et al. [8], disruption risks may lead to both negative financial effects and serious operational consequences, i.e. higher transportation costs, delays in delivery of orders, inventory shortages, loss of market shares, etc. Therefore, it is important to incorporate disruption risks carefully when configuring supply chain networks. Most studies have focused on managing disruption risks in the facility location problems, while there is a less attention on incorporating this type of risks when designing a forward-reverse logistics network.

One of the first reliability models refers to the unreliable $p$-median and $(p, q)$-center location problem, in which suppliers may be inactive by a given probability [24]. Snyder and Daskin [25] formulate the random disruptions of a facility in a facility reliability problem by a $r$-level assignment approach. According to this approach, a customer will be served if and only if all of the assigned facilities at levels $0,1, \cdots, r-1$ fail. In addition, the same disruption probability is considered for all distribution centers. Several reliability models, similar to that presented by Snyder and Daskin [25], have been developed in the literature, but the uniformdisruption-probability assumption is relaxed using a variety of modeling approaches [8,26-31].

Lim et al. [31] introduced a hardening strategy and incorporated it into a mixed integer programming model to hedge the impact of random facility disruptions. Two types of facilities, i.e. reliable facilities that are not subject to disruptions but are the more expensive and unreliable ones, which may be disrupted, are considered in their proposed model. In the hardening strategy, the reliable facilities are protected against random disruptions by a substantial investment and therefore, disruptions cannot affect them. Azad et al. [32] extend the hardening strategy introduced by Lim et al. [31] and propose the soft hardening strategy for a supply chain network design under random facility disruptions. Almost in all studies pertaining to reliable facility location problem, except Azad et al. [32], it is assumed that facilities may fully fail by a disruptive event and thus, they may not service their assigned customers. Furthermore, the capacity restrictions and disruptions are not considered in these studies. However, in real world, facilities may lose a portion of their capacities after disruptions. In this regard, Azad et al. [32] suppose that the capacity of unreliable facilities may be partially disrupted. Furthermore, it is assumed that reliable facilities have unlimited capacity while unreliable ones have finite capacities. Besides, Davarzani et al. [33] discussed the effect of single/dual/multiple sourcing to handle the potential disruptions occurred in supply chains. Lim et al. [34] consider a facility location problem in the presence of random 
facility disruptions where facilities can be protected with additional investments. Whereas most existing models in the literature implicitly assume that the disruption probability estimate is perfectly accurate, the authors investigated the impact of misestimating the disruption probability. Furthermore, Aboolian et al. [35] studied the reliable facility location problems in which facilities are subject to unexpected failures, and customers may be reassigned to facilities other than their regular facilities. The objective of these problems is to minimize the total expected costs in normal and failure scenarios.

A stochastic mathematical formulation is proposed for designing a network of multi-product supply chains comprising several capacitated production facilities, distribution centers, and retailers in markets under demand-side and supply-side uncertainties. The supply-side uncertainty includes possible disruptions in manufacturers, distribution centers, and the connecting links of the network [36]. Babazadeh and Razmi [37] present an efficient Mixed Integer Linear Programming (MILP) model that is able to consider the key characteristics of agile supply chain, which is the best competitive strategy for high turbulent environments, such as direct shipments, outsourcing, different transportation modes, discount, alliance (process and information integration) between the opened facilities and the maximum waiting time of customers for deliveries. Additionally, the robust stochastic programming approach is applied to handle both operational and disruption risks of the agile supply chain network. Garcia-Herreros et al. [38] proposed a twostage stochastic programming framework to design supply chains under the risk of facility disruptions by simultaneously considering decisions on the facility location and the inventory management.

However, the current literature on forward-reverse logistics network design with reliability consideration is relatively limited. In this line of research, Vahdani et al. [39-41] propose various models for reliable design of a closed-loop logistics network in an iron and steel industry. Various solution approaches are proposed to solve the concerned problem. In these studies, the reliability concepts are considered for the collection centers with unlimited capacities. The $r$ level assignment approach introduced by Snyder and Daskin [25] is also adopted to deal with disruptions occurred at collection centers. Hatefi and Jolai [42] introduced a scenario based model for designing an integrated forward-reverse logistics network in which the customer demand is considered as an uncertain parameter and facilities are subject to the threat of disruptions. A scenario planning approach and a $p$ robustness criterion are developed to handle facility disruptions and control reliability of the network. Their proposed model can just protect the logistic networks against complete facility disruptions. To deal with this problem, Hatefi and Jolai [43] utilized a scenario planning approach and a robust optimization developed by Bertsimas and Sim [44,45] to model both partial and complete facility disruptions. In this paper, we introduce several reliability strategies to mitigate the impacts of disruptions.

This paper offers a mixed integer linear programming for robust and reliable design of an integrated forward-reverse logistics network where facilities may be randomly disrupted, and network parameters are uncertain. Our main contributions, which distinguish our work from those of relevant published works, are listed as follow:

- Offering a robust and reliable model for designing a capacitated forward-reverse logistics network with hybrid facilities, which can tackle random facility disruptions as well as the uncertainties embedded in the input data;

- Considering random disruptions at hybrid facilities, which play a critical role in the forward and reverse flows, concurrently;

- Imposing capacity restrictions on hybrid facilities and other facilities embedded in the concerned logistics network;

- Incorporating two reliability strategies in the developed model:

- Locating two types of facilities, namely, reliable and unreliable hybrid ones;

- Employing a sharing strategy which allows products to be shipped from reliable hybrid facilities to unreliable ones for their lost capacities to be compensated.

- Considering partial and complete capacity disruptions at unreliable hybrid facilities. The capacity of unreliable facilities may be lost partially due to the threat of disruptions. Therefore, they can serve their customers by the remaining of their available capacities;

- Applying robust optimization approach to handle uncertainties in input data, i.e. demands, returned products, fixed opening costs, and capacities.

The rest of the paper is organized as follows. In Section 2, the studied problem is defined and the proposed reliability-based model is elaborated. In Section 3, the robust optimization approach is briefly explained and the robust counterpart of the proposed reliabilitybased model is developed. Several computational experiments and related numerical results along with a sensitivity analysis are reported in Section 4. Finally, concluding remarks are discussed in Section 5 . 


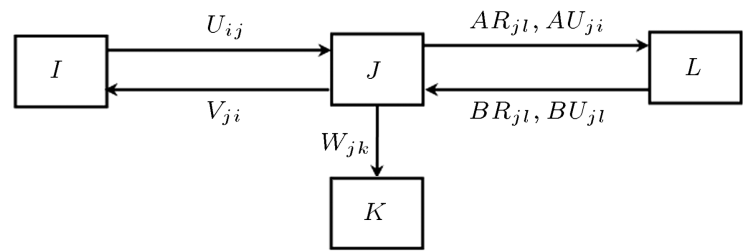

Figure 1. Integrated forward-reverse logistics network.

\section{Problem definition and formulation}

We consider a single product, multi-echelon forwardreverse logistics network consisting of production and distribution centers with limited capacities in the forward flow and collection, recovery and disposal centers with limited capacities in the reverse flow. The Hybrid Production-Recovery (HPR) and Hybrid DistributionCollection (HDC) facilities are considered in the Integrated Forward-Reverse Logistics (IFRL) network, which has several advantages such as cost saving and pollution reduction $[2,4,46]$. The HPR facilities play the role of production centers in the forward flow and recovery centers in the reverse flow. Similarly, HDC facilities act as the distribution and collection centers in the forward and reverse flows, respectively. The structure of the concerned IFRL network is depicted in Figure 1.

As it is depicted in Figure 1, new products are shipped from HPR centers to customer zones through HDC centers in order to satisfy customer demands in the forward flow. In the reverse flow, the returned products are collected in the HDC centers for inspection purpose. After testing, they are divided into the recoverable and scrap products. The recoverable products are shipped to the HPR centers for recovery processes. Then, they are entered to the forward network as new products. The scrap products are shifted to the disposal centers. The average disposal rate reflects the quality of returned products; since high quality returns have a capability for recovery process (remanufacturing and de-manufacturing) and low quality returns must be entered to a safe disposal process. It is also assumed that the customer zones are predefined and fixed. The goal is finding the optimal number of required facilities (i.e., HPR, HDC, and disposal centers) and their best locations as well as the optimal quantity of material flows between them, while minimizing the total costs of the designed network. Gaining this goal highly depends on the way that uncertainty and reliability issues are handled when configuring the IFRL network. HPR and HDC facilities play an important role in both forward and reverse networks. However, for the sake of simplicity and without loss of generality, we assume that just HDC facilities are subject to random disruptions. Therefore, the reliability issues are taken into account for HDC facilities. However, similar reliability concepts can be considered for HPR facilities when they are subject to the threat of disruptions.

At each node, $j$, an unreliable HDC facility can be located at a fixed cost of $\tilde{F} U_{j}$, which may fail with probability:

$$
q_{j}\left(0<q_{j}<1\right)
$$

or a reliable HDC facility at a cost of $\tilde{F} R_{j}$ which never fails. Disruptions occur at unreliable facilities, while reliable facilities are protected against disruptions. The reliable HDC facilities never fail, since we can strengthen them against disruptions by a financial investment and hence, disruptions do not affect them. In other words, the network can be made significantly more reliable to disruptions with additional investment in the infrastructure, which makes planning for disruptions more attractive from a managerial prospective. Obviously, the cost of opening reliable HDC facilities is more than that of unreliable ones (i.e., $\tilde{F} R_{j}>$ $\left.\tilde{F} U_{j} ; \forall j\right)$. At the HDC facility, $j$, the distribution and collection capacities are denoted by $\tilde{\gamma}_{j}$ and $\tilde{\eta}_{j}$, respectively. The previous studies addressing the facility reliability problem assume unlimited capacities, while we consider a capacitated IFRL network model. Furthermore, these studies suppose that the disrupted facilities completely fail after disruptions and cannot serve their assigned customers. However, we assume unreliable HDC facilities may lose a part of their distribution/collection capacities when a disruption strikes. Therefore, in the forward flow, they can serve the assigned customers with the remaining of their available distribution capacities. Notably, in the reverse flow, they can serve the HPR and disposal centers with the rest of their collection capacities. In this regard, the capacity failure fraction is defined for an unreliable HDC facility, which shows the percentage of the lost capacity during disruption situations. The notations $p_{j}$ and $p_{j}^{\prime}$, respectively, denote the percentages of distribution and collection capacities at unreliable HDC facility, $j$, which are lost as a result of disruption.

A sharing strategy is also considered in the forward flow, which allows reliable HDC facilities to share new products to unreliable HDC facilities to compensate their lost capacities. In this manner, new products can be trans-shipped from reliable HDC facilities to unreliable ones whose capacities have been partially disrupted. Therefore, customers of a disrupted HDC center are not necessarily assigned to other HDC centers, since the lost capacity of a disrupted HDC center will be amended by reliable HDC facilities. It is worthy to mention that without loss of generality, the sharing strategy is only applied on distribution capacity of HDC facilities in the forward flow. To develop the mathematical programming model, the following notations are used. 


\subsection{Notations}

\section{Sets:}

I : $\quad$ Number of potential HPR centers, indexed by $i$;

$J$ : $\quad$ Number of potential HDC centers, indexed by $j$;

$K$ : $\quad$ Number of potential disposal centers, indexed by $k$;

$L$ : $\quad$ Number of fixed customer zones, indexed by $l$.

\section{Parameters:}

$d_{l}: \quad$ Demand of customer zone $l$;

$r_{l}: \quad$ Amount of returned products from customer zone $l$;

Ad : $\quad$ Average disposal fraction;

$c_{a b}: \quad$ Transportation cost per unit of products from a center $a$ to a center $b$ or a customer zone $b$ for $a, b \in I, J, K, L$;

$\varphi_{i}: \quad$ Production capacity of HPR center $i$ in the forward flow;

$\tau_{i}: \quad$ Recovery capacity of HPR center $i$ in the reverse flow;

$\gamma_{j}$ : $\quad$ Distribution capacity of HDC center $j$ in the forward flow;

$\theta_{j}: \quad$ Collection capacity of HDC center $j$ in the reverse flow;

$\omega_{k}: \quad$ Disposal capacity of disposal center $k$;

$F_{i}: \quad$ Fixed cost of opening HPR center $i$;

$F R_{j}$ : $\quad$ Fixed cost of opening reliable HDC center $j$;

$F U_{j}$ : $\quad$ Fixed cost of opening unreliable HDC center $j$;

$F D_{k}: \quad$ Fixed cost of opening disposal center $k$;

$c p f_{i}$ : $\quad$ Production cost per unit of product at HPR center $i$;

$c p r_{i}$ : $\quad$ Recovery cost per unit of product at HPR center $i$;

$c d f_{j}$ : $\quad$ Distribution cost per unit of product at $\mathrm{HDC}$ center $j$;

$c d r_{j}$ : Collection cost per unit of product at HDC center $j$;

$c p_{k}$ : Disposal cost per unit of scrapped product at disposal center $k$;

$q_{j}$ : Disruption probability in unreliable HDC center $j$;

$p_{j}: \quad$ Percentage of disrupted distribution capacity at opened unreliable HDC center $j$; $p_{j}^{\prime}$ : $\quad$ Percentage of disrupted collection capacity at opened unreliable HDC center $j$.

Variables:

$U_{i j}$ : Quantity of products shipped from HPR center $i$ to HDC center $j$;

$V_{j i}: \quad$ Quantity of recoverable products shipped from HDC center $j$ to HPR center $i$;

$W_{j k}$ : Quantity of scrapped products shipped from HDC center $j$ to disposal center $k$;

$T_{j^{\prime} j}$ : Quantity of products trans-shipped from reliable HDC center $j^{\prime}$ to unreliable HDC center $j$ at a disrupted situation $\left(j^{\prime} \neq j\right)$;

$X_{i}$ : $\quad$ Binary variable; equals 1 if HPR center $i$ is opened, 0 otherwise;

$Y R_{j}$ : $\quad$ Binary variable; equals 1 if reliable HDC center $j$ is opened, 0 otherwise;

$Y U_{j}$ : $\quad$ Binary variable; equals 1 if unreliable HDC center $j$ is opened, 0 otherwise;

$Z_{k}$ : $\quad$ Binary variable; equals 1 if disposal center $k$ is opened, 0 otherwise;

$A R_{j l}$ : $\quad$ Binary variable; equals 1 if customer zone $l$ is assigned to reliable HDC center $j$ in the forward flow, 0 otherwise;

$A U_{j l}$ : $\quad$ Binary variable; equals 1 if customer zone $l$ is assigned to unreliable HDC center $j$ in the forward flow, 0 otherwise;

$B R_{j l}$ : $\quad$ Binary variable; equals 1 if customer zone $l$ is assigned to reliable HDC center $j$ in the reverse flow, 0 otherwise;

$B U_{j l}$ : $\quad$ Binary variable; equals 1 if customer zone $l$ is assigned to unreliable HDC center $j$ in the reverse flow, 0 otherwise.

\subsection{Problem formulation}

The developed model is a mixed integer linear programming model, which is formulated as follows:

$$
\begin{aligned}
& P(\mathrm{I}): \min \sum_{i} F_{i} X_{i}+\sum_{j} F R_{j} Y R_{j}+\sum_{j} F U_{j} Y U_{j} \\
& +\sum_{k} F D_{k} Z_{k}+\sum_{i} \sum_{j}\left(c_{i j}+c p f_{i}\right) U_{i j} \\
& \quad+\sum_{j} \sum_{l}\left(c_{j l}+c d f_{j}\right) d_{l}\left(A R_{j l}+A U_{j l}\right)
\end{aligned}
$$




$$
\begin{aligned}
& +\sum_{l} \sum_{j}\left(c_{l j}+c d r_{j}\right) r_{l}\left(B R_{l j}+B U_{l j}\right) \\
& +\sum_{j} \sum_{k}\left(c_{j k}+c p_{k}\right) W_{j k}+\sum_{j} \sum_{i}\left(c_{j i}+c p r_{i}\right) V_{j i} \\
& +\sum_{j^{\prime}} \sum_{j \neq j^{\prime}} q_{j} c_{j^{\prime} j} T_{j^{\prime} j}, \\
& \text { s.t. } \sum_{j} A R_{j l}+\sum_{j} A U_{j l}=1 \quad \forall l, \\
& \begin{array}{l}
\sum_{j} B R_{l j}+\sum_{j} B \\
\sum_{j} Y R_{j} \geq 1,
\end{array} \\
& \forall l, \\
& Y R_{j}+Y U_{j} \leq 1 \quad \forall j \\
& A R_{j l} \leq Y R_{j} \quad \forall j, l, \\
& B R_{l j} \leq Y R_{j} \quad \forall j, l, \\
& T_{j^{\prime} j} \leq M Y R_{j^{\prime}} \quad \forall j^{\prime}, j \neq j^{\prime}, \\
& T_{j^{\prime} j} \leq \gamma_{j} Y U_{j} \quad \forall j^{\prime}, j \neq j^{\prime}, \\
& \sum_{j^{\prime}} T_{j^{\prime} j}+\gamma_{j}\left(1-p_{j}\right) Y U_{j} \geq \sum_{l} d_{l} A U_{j l} \quad \forall j, \\
& \sum_{j} T_{j^{\prime} j}+\sum_{l} d_{l} A R_{j^{\prime} l} \leq \gamma_{j^{\prime}} Y R_{j^{\prime}} \\
& \sum_{i} U_{i j}+\sum_{j^{\prime}} T_{j^{\prime} j} \geq \sum_{l} d_{l} A U_{j l} \\
& \sum_{i} V_{j i}=\sum_{l}(1-A d) r_{l}\left(B R_{l j}+B U_{l j}\right) \quad \forall j, \\
& \sum_{k} W_{j k}=\sum_{l} A d r_{l}\left(B R_{l j}+B U_{l j}\right) \quad \forall j, \\
& \sum_{i} \sum_{j} U_{i j} \geq \sum_{l} d_{l} \\
& \sum_{j} U_{i j} \leq \varphi_{i} X_{i} \quad \forall i \\
& \sum_{j} V_{j i} \leq \tau_{i} X_{i} \quad \forall i \\
& \sum_{i} U_{i j} \leq \gamma_{j}\left(Y U_{j}+Y R_{j}\right) \quad \forall j, \\
& \sum_{l} d_{l} A U_{j l} \leq \gamma_{j} Y U_{j} \quad \forall j
\end{aligned}
$$

$$
\begin{aligned}
& \sum_{l} r_{l} B U_{l j} \leq\left(1-p_{j}^{\prime}\right) \theta_{j} Y U_{j} \\
& \sum_{l} r_{l} B R_{l j} \leq \theta_{j} Y R_{j} \\
& \sum_{j} W_{j k} \leq \omega_{k} Z_{k} \\
& X_{i}, Y R_{j}, Y U_{j}, Z_{k}, A R_{j l}, A U_{j l}, B R_{l j}, B U_{j l} \in\{0,1\} \\
& \forall i \in I, \forall j \in J, \forall l \in L, \forall k \in K, \\
& U_{i j}, W_{j k}, V_{j i}, T_{j^{\prime} j} \geq 0, \\
& \forall i \in I, \forall j, j^{\prime} \in J, \forall l \in L, \forall k \in K,
\end{aligned}
$$

where $M$ is a large positive number. The aim of the objective function of Relation (1) is to minimize the total costs, including the fixed costs of opening facilities, processing and transportation costs, and the expected costs of sharing products from reliable HDC facilities to unreliable HDC facilities. The 1st to the 4 th terms show the costs of locating HPR facilities, reliable and unreliable HDC facilities, and disposal centers, respectively. The 5 th term represents transportation costs from HPR to HDC centers and production processing costs at HPR centers. The 6th term indicates the costs of assigning customers to reliable and unreliable HDC centers in the forward network, respectively, and distribution processing costs at HDC facilities. The 7 th term presents the costs of assigning customers to reliable and unreliable HDC centers in the reverse flow, respectively, and collection processing costs at HDC facilities. The 8 th and 9th terms show the transportation costs from HDC centers to disposal and HPR centers, and the disposal and recovery processing costs at disposal and HPR facilities, respectively. The last term presents the expected disruption costs, which is the expected cost of sharing products from reliable HDC centers to reliable HDC centers during disruptions.

Constraints (2) and (3) ensure that each customer zone should be exactly assigned to one HDC facility in the forward and reverse flows, respectively. Constraint (4) shows that at least one reliable HDC center must be opened to enforce the sharing strategy in a disruption situation. Constraint (5) indicates that both reliable and unreliable HDC facilities cannot be opened simultaneously at a potential node $j$. Constraints (6) and (7) enforce the creation of a reliable HDC center at potential node $j$, if a customer is assigned to it in both forward and reverse flows. Constraint (8) guarantees that in a disruption situation, if a reliable HDC facility is located at potential node $j^{\prime}$, products can be trans-shipped from it to unreliable HDC facilities. Constraint (9) ensures that during disruption 
situation, products cannot be shipped to potential node $j$, unless an unreliable HDC facility is located at it. Constraint (10) states that for an unreliable HDC center located at node $j$, the sum of products trans-shipped from reliable HDC facilities to it and its available capacity after disruption should be greater than or equal to the total demands of its assigned customers.

Constraint (11) indicates that for a reliable HDC center opened at potential node $j^{\prime}$, the sum of products trans-shipped from this facility to unreliable HDC facilities and the total demands of its assigned customers should not exceed its capacity. Constraint (12) states that the sum of flows entered to an unreliable HDC center located at node $j$ cannot be lower than the total demands of its assigned customers. Constraints (13) to (15) address the balance constraints. Constraints (16) and (17) enforce restrictions on the production and recovery capacities at HPR centers, respectively. Constraints (18) to (21) enforce restrictions on the distribution and collection capacities at HDC facilities in the forward and reverse flows, respectively. Notably, the lost distribution capacity occurred at unreliable HDC centers can be amended by the means of sharing strategy which is integrated in the forward flow. This issue is reflected in Constraints (10) and (19). Constraint (22) enforces the capacity restrictions at disposal centers. Finally, Constraints (23) and (24) denote the binary and flow variables and their corresponding restrictions.

\section{Robust optimization model}

Soyster [47] is the first who introduces the idea of Robust Optimization (RO), but his idea turns to be very pessimistic, which makes it unfavorable among practitioners. Furthermore, the RO approach was developed independently by El-Ghaoui and Lebert [48], ElGhaoui et al. [49], and Ben-Tal and Nemirovsky [50,51]. The Ben-Tal and Nemirovsky approach [50,51] provides less conservative solutions than earlier worst-case solutions provided by robust mathematical programming approaches (e.g., [47]) by trading off some of the conservatism for improvement in the objective function by bounding the set of values uncertain parameters could achieve. A key feature of the RO approach is its tractability, which depends on the structure of the uncertainty set. Bertsimas and Sim [44,45] develop different robust optimization techniques in an attempt to keep the structure of the original problem. The optimal solution is more optimistic than the robust optimization approach introduced by Soyster [47]. Furthermore, Bertsimas and Sim [44,45] develop a new robust methodology, wherein the optimal solution is more optimistic. In this paper, we follow the robust optimization approach developed by Ben-Tal and Ne- mirovski [50-52]. The significant advantages of this approach are as follow:

a) The robust formulation of the problem is tractable when the uncertainty set is described as a box or an ellipsoid. Furthermore, the structure of the final robust method remains the same as the nominal problem in the case of box uncertainty;

b) It is less conservative rather than the robust optimization introduced by Soyster [47] and Bertsimas and Sim [44,45] which makes it more favorable;

c) It allows us to control the level of conservatism in the solution set by changing uncertainty level.

Nowadays, robust optimization method is embedded into the mathematical programming problems to deal with the uncertainty in the model parameters. Robust optimization technique could provide a solution that is guaranteed to be good for all or most of the possible realizations of the uncertain parameters. To explain the robust optimization technique, consider the following linear programming problem, in which the parameters $c, A$, and $b$ belong to a given uncertainty set $U$ :

$$
\begin{aligned}
& \text { Min } c x, \\
& \text { s.t. } A x \leq b,
\end{aligned}
$$

where $x$ is the vector of decision variable. The concerned uncertain linear optimization model contains a series of linear optimization models whose parameters vary in the uncertainty set $U$ [50-53]. According to Ben-Tal and Nemirovski [50-53], the robust counterpart (RO) of the model (Relation (25)) can be formulated as follows:

$$
\operatorname{Min}\left\{\sup _{(c, d, A, b \in U)}[c x]: A x \leq b, \forall c, d, A, b \in U\right\} .
$$

A vector $x$ is a 'robust feasible' solution, if it satisfies all realizations of the constraints according to the uncertainty set $U$. Furthermore, it is a 'robust optimal' solution when there is no other feasible solution with better objective function value. In the case where uncertainty set is in the form of box uncertainty ( $\left.U_{\text {box }}\right)$, the robust counterpart can be converted to a tractable equivalent model in which the extreme points of the box uncertainty are substituted instead of $U_{\text {box }}$ [54]. Under box uncertainty, each uncertain parameter such as $\tilde{a}_{i j}$ is unknown, but bounded in a box of the form $U_{\text {box }}=\left\{a_{i j} \in R:\left|a_{i j}-\bar{a}_{i j}\right| \leq \rho_{a} G_{i j}^{a}\right\}$, where $\bar{a}_{i j}, \rho_{a}$, and $G_{i j}^{a}$ denote the nominal value, the uncertainty level, and scale uncertainty, respectively. Notably, $G_{i j}^{a}$ is a positive number and $0<\rho_{a} \leq 1$. If we set $G_{i j}^{a}=a_{i j}$, then the relative deviation from the nominal value is at most $\rho_{a}$. According to Ben-Tal et al. [54] 
and Pishvaee et al. [16], the tractable equivalent model of the RO problem (26) can be written as follows:

$$
\begin{aligned}
& \operatorname{Min} \quad z, \\
& \text { s.t. } \quad \sum_{j}\left(\bar{c}_{j} x_{j}+\eta_{j}\right) \leq z, \\
& \rho_{c} G_{j}^{c} \leq \eta_{j}, \quad \forall j, \\
& \rho_{c} G_{j}^{c} \geq-\eta_{j}, \quad \forall j, \\
& \sum_{j}\left(\bar{a}_{i j}+\rho_{a} G_{i j}^{a}\right) x_{j} \leq \bar{b}_{i}-\rho_{b} G_{i}^{b}, \quad \forall i .
\end{aligned}
$$

The supply chain decisions can be categorized into three types according to their significance and the length of the planning horizon considered. First, decisions associated with the place, volume, and technology of facilities are often regarded as strategic with a planning horizon of several years. Second, supplier evaluation and selection, distribution channel, and transportation mode selection are the tactical decisions which can change every few months. Finally, operational decisions that are easily revised in the short term contain the decisions about raw material and semi-finished and finished product flows in the supply chain network. There are both single-period and multiperiod supply chain network design problems in the concerned literature. Multi-period planning horizon models have been proposed for situations in which parameters change over time in a predictable way. Thereby, a planning horizon, divided into several time periods, is usually considered [55]. However, the proposed model is a single-period planning horizon model in which some parameters are uncertain. To develop the robust counterpart of the proposed reliability model and its tractable form, it is supposed that opening costs $\left(\tilde{F}_{i}, \tilde{F} R_{j}, \tilde{F} U_{j}, \tilde{F} D_{k}\right)$, demands $\left(\tilde{d}_{l}\right)$, returned products $\left(\tilde{r}_{l}\right)$, and capacities $\left(\tilde{\varphi}_{i}, \tilde{\tau}_{i}, \tilde{\gamma}_{j}, \tilde{\theta}_{j}, \tilde{\omega}_{k}\right)$ are uncertain and can vary in their corresponding box uncertainty sets.

Consequently, the robust counterpart of the proposed model can be formulated as the following equivalent tractable model:

$$
\begin{aligned}
& P(\mathrm{II}): \min z \sum_{i}\left(\bar{F}_{i} X_{i}+\eta_{i}^{F}\right) \\
& +\sum_{j}\left(\bar{F} R_{j} Y R_{j}+\eta_{j}^{F R}\right)+\sum_{j}\left(\bar{F} U_{j} Y U_{j}+\eta_{j}^{F U}\right) \\
& \quad+\sum_{k}\left(\bar{F} D_{k} Z_{k}+\eta_{k}^{F D}\right)+\sum_{i} \sum_{j}\left(c_{i j}+c p f_{i}\right) U_{i j} \\
& \quad+\sum_{j} \sum_{l}\left(c_{j l}+c d f_{j}\right)\left[\bar{d}_{l}\left(A R_{j l}+A U_{j l}\right)+\eta_{l}^{d}\right]
\end{aligned}
$$

$$
\begin{aligned}
& +\sum_{l} \sum_{j}\left(c_{l j}+c d r_{j}\right)\left[\bar{r}_{l}\left(B R_{l j}+B U_{l j}\right)+\eta_{l}^{r}\right] \\
& +\sum_{j} \sum_{k}\left(c_{j k}+c p_{k}\right) W_{j k}+\sum_{j} \sum_{i}\left(c_{j i}+c p r_{i}\right) V_{j i} \\
& +\sum_{j^{\prime}} \sum_{j \neq j^{\prime}} q_{j} c_{j^{\prime} j} T_{j^{\prime} j} \leq z
\end{aligned}
$$

s.t. $\rho_{F} G_{i}^{F} X_{i} \leq \eta_{i}^{F} \quad \forall i$,

$\rho_{F} G_{i}^{F} X_{i} \geq-\eta_{i}^{F} \quad \forall i$,

$\rho_{F R} G_{j}^{F R} Y R_{j} \leq \eta_{j}^{F R} \quad \forall j$,

$\rho_{F R} G_{j}^{F R} Y R_{j} \geq-\eta_{j}^{F R} \quad \forall j$,

$\rho_{F U} G_{j}^{F U} Y U_{j} \leq \eta_{j}^{F U} \quad \forall j$,

$\rho_{F U} G_{j}^{F U} Y U_{j} \geq-\eta_{j}^{F U} \quad \forall j$,

$\rho_{F D} G_{k}^{F D} Z_{k} \leq \eta_{k}^{F D} \quad \forall k$,

$\rho_{F D} G_{k}^{F D} Z_{k} \geq-\eta_{k}^{F D} \quad \forall k$,

$\rho_{d} G_{l}^{d}\left(A R_{j l}+A U_{j l}\right) \leq \eta_{l}^{d} \quad \forall j, l$,

$\rho_{d} G_{l}^{d}\left(A R_{j l}+A U_{j l}\right) \geq-\eta_{l}^{d} \quad \forall j, l$,

$\rho_{r} G_{l}^{r}\left(B R_{l j}+B U_{l j}\right) \leq \eta_{l}^{r} \quad \forall l, j$,

$\rho_{r} G_{l}^{r}\left(B R_{l j}+B U_{l j}\right) \geq-\eta_{l}^{r} \quad \forall l, j$,

$\sum_{j} A R_{j l}+\sum_{j} A U_{j l}=1 \quad \forall l$,

$\sum_{j} B R_{l j}+\sum_{j} B U_{l j}=1 \quad \forall l$,

$\sum_{j} Y R_{j} \geq 1$

$Y R_{j}+Y U_{j} \leq 1 \quad \forall j$,

$A R_{j l} \leq Y R_{j} \quad \forall j, l$,

$B R_{l j} \leq Y R_{j} \quad \forall j, l$,

$T_{j^{\prime} j} \leq M Y R_{j^{\prime}} \quad \forall j^{\prime}, j \neq j^{\prime}$,

$T_{j^{\prime} j} \leq\left(\bar{\gamma}_{j}-\rho_{\gamma} G_{j}^{\gamma}\right) Y U_{j} \quad \forall j^{\prime}, j \neq j^{\prime}$,

$\sum_{j^{\prime}} T_{j^{\prime} j}+\left(\bar{\gamma}_{j}-\rho_{\gamma} G_{j}^{\gamma}\right)\left(1-p_{j}\right) Y U_{j}$

$$
\geq \sum_{l}\left(\bar{d}_{l}+\rho_{d} G_{l}^{d}\right) A U_{j l} \quad \forall j
$$




$$
\begin{aligned}
& \sum_{j} T_{j^{\prime} j}+\sum_{l}\left(\bar{d}_{l}+\rho_{d} G_{l}^{d}\right) A R_{j^{\prime} l} \\
& \leq\left(\bar{\gamma}_{j^{\prime}}-\rho_{\gamma} G_{j^{\prime}}^{\gamma}\right) Y R_{j^{\prime}} \quad \forall j^{\prime} \\
& \sum_{i} U_{i j}+\sum_{j^{\prime}} T_{j^{\prime} j} \geq \sum_{l}\left(\bar{d}_{l}+\rho_{d} G_{l}^{d}\right) A U_{j l} \quad \forall j \\
& \sum_{i} V_{j i}=\sum_{l}(1-A d)\left(\bar{r}_{l}+\rho_{r} G_{l}^{r}\right)\left(B R_{l j}+B U_{l j}\right) \quad \forall j \\
& \sum_{k} W_{j k}=\sum_{l} A d\left(\bar{r}_{l}+\rho_{r} G_{l}^{r}\right)\left(B R_{l j}+B U_{l j}\right) \quad \forall j \\
& \sum_{i} \sum_{j} U_{i j} \geq \sum_{l}\left(\bar{d}_{l}+\rho_{d} G_{l}^{d}\right) \\
& \sum_{j} U_{i j} \leq\left(\bar{\varphi}_{i}-\rho_{\varphi} G_{i}^{\varphi}\right) X_{i} \quad \forall i \\
& \sum_{j} V_{j i} \leq\left(\bar{\tau}_{i}-\rho_{\tau} G_{i}^{\tau}\right) X_{i} \quad \forall i \\
& \sum_{i} U_{i j} \leq\left(\bar{\gamma}_{j}-\rho_{\gamma} G_{j}^{\gamma}\right)\left(Y U_{j}+Y R_{j}\right) \\
& \sum_{l}\left(\bar{d}_{l}+\rho_{d} G_{l}^{d}\right) A U_{j l} \leq\left(\bar{\gamma}_{j}-\rho_{\gamma} G_{j}^{\gamma}\right) Y U_{j} \\
& \sum_{l}\left(\bar{r}+\rho_{r} G_{l}^{r}\right) B U_{l j} \leq\left(1-p_{j}^{\prime}\right)\left(\bar{\theta}_{j}-\rho_{\theta} G_{j}^{\theta}\right) Y U_{j} \forall j \\
& \sum_{l}\left(\bar{r}_{l}+\rho_{r} G_{l}^{r}\right) B R_{l j} \leq\left(\bar{\theta}_{j}-\rho_{\theta} G_{j}^{\theta}\right) Y R_{j} \forall j \\
& \sum_{j} W_{j k} \leq\left(\bar{\omega}_{k}-\rho_{\omega} G_{k}^{\omega}\right) Z_{k} \quad \forall k \\
& X_{i}, Y R_{j}, Y U_{j}, Z_{k}, A R_{j l}, A U_{j l}, B R_{l j}, B U_{j l} \in\{0,1\} \\
& \forall i \in I, \forall j \in J, \forall l \in L, \forall k \in K \\
& U_{i j}, W_{j k}, V_{j i}, T_{j^{\prime} j}, \eta_{i}^{F}, \eta_{j}^{F R}, \eta_{j}^{F U}, \eta_{k}^{F D}, \eta_{l}^{d}, \eta_{l}^{r} \geq 0 \\
& \forall i \in I, \forall j, j^{\prime} \in J, \forall l \in L, \forall k \in K
\end{aligned}
$$

\section{Computational experiments and sensitivity analysis results}

In this section, several numerical experiments are conducted to show the significance and performance of the proposed deterministic and robust models, i.e. $P(\mathrm{I})$ and $P(\mathrm{II})$, respectively. To this end, two test problems, whose details are presented in Table 1 , are taken into account and the results are provided under four uncertainty levels, i.e. $\rho=0.25,0.5,0.75,1$. Furthermore, nominal data is randomly generated from the uniform distributions presented in Table 2.

To provide numerical results, the deterministic and robust models are first solved under nominal data. Then, under each uncertainty level, five random realizations are generated from the corresponding uncertainty set (i.e., $\sim$ [nominal value $\rho_{\bullet} G_{\bullet}^{*}$, nominal value $\left.\left.+\rho_{\bullet} G_{\bullet}^{*}\right]\right)$ to investigate the behavior of the solutions provided by the proposed deterministic and robust models. The models can update their tactical decision variables, namely, flow quantities between facilities, i.e. the continuous variables, and assignment variables indicating the assignment of customer zones to the hybrid facilities under each realization. Due to the strategic nature of the decisions regarding the number and location of facilities, and since they cannot be changed in the short time $[16,56]$, the corresponding location variables are fixed and cannot be changed under various realizations. However, the violation of chance constraints under realizations should be considered as a penalty in the objective function of the deterministic and robust models [57]. Both models are coded in GAMS 23.5/CPLEX 12.2 optimization software and all numerical experiments are solved using a Pentium dual-core $2.10 \mathrm{GHz}$ computer with 3 GB RAM.

The deterministic and robust models are first solved under nominal data. Notably, all uncertainty levels, i.e. $\rho_{F}=\rho_{F R}=\rho_{F U}=\rho_{F D}=\rho_{d}=\rho_{r}=\rho_{\gamma}=$ $\rho_{\varphi}=\rho_{\tau}=\rho_{\theta}=\rho_{\omega}$, are equally varied. The respected results are reported in the third and fourth columns of Table 3. Furthermore, the computational times are also reported for two test problems in Table 3 .

According to these results, it can be affirmed that the total cost of the concerned forward-reverse network problem under uncertainty is greater than that of the respective deterministic model as expected. On the other hand, with additional costs in the infrastructure, the forward-reverse network will be significantly more stable against uncertainties. Furthermore, by augmenting the supply chain uncertainty level, total costs of the supply chain network increase due to the conservative nature of the robust optimization approach to uncertainty level.

Both deterministic and robust models are also solved under random realizations data. The mean and standard deviation of the objective function values under various realizations are considered as two performance criteria to evaluate these models. The computational results under random realizations are reported in the fifth to the eighth columns of Table 3. The entire results are also shown in Table 4 . The results presented in Table 3 affirm that the robust model generates the solution with both higher quality 
Table 1. Details of numerical experiments.

\begin{tabular}{ccccccc}
\hline $\begin{array}{c}\text { Problem } \\
\text { no. }\end{array}$ & $\begin{array}{c}\text { No. of } \\
\text { potential } \\
\text { HPR centers }\end{array}$ & $\begin{array}{c}\text { No. of } \\
\text { potential } \\
\text { HDC centers }\end{array}$ & $\begin{array}{c}\text { No. of } \\
\text { potential } \\
\text { disposal centers }\end{array}$ & $\begin{array}{c}\text { No. of } \\
\text { customer } \\
\text { zones }\end{array}$ & $\begin{array}{c}\text { No. of } \\
\text { binary } \\
\text { variables }\end{array}$ & $\begin{array}{c}\text { No. of } \\
\text { constraints }\end{array}$ \\
\hline 1 & 5 & 5 & 3 & 8 & 218 & 225 \\
2 & 7 & 10 & 5 & 15 & 632 & 631 \\
\hline
\end{tabular}

Table 2. The sources of random generation of model parameters.

\begin{tabular}{clcl}
\hline Parameter & $\begin{array}{c}\text { Related random } \\
\text { distribution }\end{array}$ & Parameter & $\begin{array}{c}\text { Related random } \\
\text { distribution }\end{array}$ \\
\hline$\tilde{d}_{l}$ & $\sim$ Uniform $(150,220)$ & $c \tilde{p} f_{i}$ & $\sim$ Uniform $(3,6)$ \\
$\tilde{r}_{l}$ & $\sim$ Uniform $(90,140)$ & $c \tilde{p} r_{i}$ & $\sim$ Uniform $(3,5)$ \\
$\tilde{\varphi}_{i}$ & $\sim$ Uniform $(550,800)$ & $c \tilde{d} f_{j}$ & $\sim$ Uniform $(1.5,4)$ \\
$\tilde{\tau}_{i}$ & $\sim$ Uniform $(300,400)$ & $c \tilde{d} r_{j}$ & $\sim$ Uniform $(1.5,3)$ \\
$\tilde{\gamma}_{j}$ & $\sim$ Uniform $(350,550)$ & $\tilde{c} p_{k}$ & $\sim$ Uniform $(2,4)$ \\
$\tilde{\eta}_{j}$ & $\sim$ Uniform $(280,400)$ & $\tilde{F}_{i}$ & $\sim$ Uniform $(320000,480000)$ \\
$\tilde{\rho}_{k}$ & $\sim$ Uniform $(150,250)$ & $\tilde{F} U_{j}$ & $\sim$ Uniform $(180000,260000)$ \\
$q_{j}$ & $\sim$ Uniform $(0.025,0.15)$ & $\tilde{F} D_{k}$ & $\sim$ Uniform $(150000,220000)$ \\
$p_{j}, p_{j}^{\prime}$ & $\sim$ Uniform $(0.1,0.5)$ & $G_{l}^{d}, G_{l}^{r}$ & $\sim$ Uniform $(10,15)$ \\
$A d$ & 0.2 & $G_{i}^{\varphi}, G_{i}^{\tau}, G_{j}^{\gamma}, G_{j}^{\theta}, G_{k}^{\omega}$ & $\sim$ Uniform $(15,25)$ \\
$\tilde{d}_{a b}$ & $\sim$ Uniform $(4,10)$ & $G_{i}^{F}, G_{j}^{F R}, G_{j}^{F U}, G_{k}^{F D}$ & $\sim$ Uniform $(5000,10000)$ \\
& & $\tilde{F} R_{j}=1.2 * \tilde{F} U_{j}$ & \\
\hline
\end{tabular}

Table 3. Computational results of comparing performances of deterministic and robust models.

\begin{tabular}{|c|c|c|c|c|c|c|c|}
\hline \multirow[t]{2}{*}{$\begin{array}{l}\text { Test } \\
\text { problem } \\
\text { no. }\end{array}$} & \multirow[t]{2}{*}{$\begin{array}{c}\text { Uncertainty } \\
\text { level }\end{array}$} & \multicolumn{2}{|c|}{$\begin{array}{c}\text { Objective function } \\
\text { value under } \\
\text { nominal data }\end{array}$} & \multicolumn{2}{|c|}{$\begin{array}{l}\text { Mean of objective } \\
\text { function values } \\
\text { under realizations }\end{array}$} & \multicolumn{2}{|c|}{$\begin{array}{c}\text { Standard deviation } \\
\text { of objective } \\
\text { function values } \\
\text { under realizations }\end{array}$} \\
\hline & & $\begin{array}{c}\text { Deterministic } \\
\text { (CPU time) }\end{array}$ & $\begin{array}{c}\text { Robust } \\
\text { (CPU time) }\end{array}$ & Deterministic & Robust & Deterministic & Robust \\
\hline \multirow{4}{*}{1} & 0.25 & 2065415.7 (1.19) & $2112869.3(1.41)$ & 2068861.6 & 2096922.0 & 5903.1 & 2127.9 \\
\hline & 0.5 & & $2129360.6(1.63)$ & 2068132.0 & 2093077.5 & 5381.8 & 2240.6 \\
\hline & 0.75 & & $2146656.8(1.56)$ & 2077768.0 & 2099923.3 & 14351.3 & 6589.4 \\
\hline & 1 & & $2163368.6(0.90)$ & 2078139.7 & 2099571.9 & 25035.0 & 8727.8 \\
\hline \multirow{4}{*}{2} & 0.25 & $3413102.3(3.01)$ & $3482435.8(3.25)$ & 3392808.6 & 3408827.1 & 12223.6 & 6741.5 \\
\hline & 0.5 & & $3674012.4(3.42)$ & 3416929.0 & 3568150.6 & 8209.6 & 5049.9 \\
\hline & 0.75 & & $3707211.2(3.21)$ & 3457480.9 & 3563381.9 & 32151.6 & 11353.6 \\
\hline & 1 & & $3780839.3(3.37)$ & 3505673.8 & 3656574.5 & 38294.0 & 3101.5 \\
\hline
\end{tabular}

and lower standard deviation. Furthermore, in two test problems except test problem 2 with uncertainty level of 0.25 , the robust model dominates the deterministic one in terms of the mean of objective function values. Moreover, with respect to standard deviation, the robust approach dominates the deterministic one with a high difference in two test problems. Finally, by comparing the columns 4 and 6 in Table 3 , it can be concluded that the mean values of the objective function obtained by the robust optimization model under realizations are lower than those provided under nominal values. The reason for this matter can be interpreted as follows. The robust optimization protects the network against the worst case values of uncertain input data. In this manner, the total cost of the network (i.e., the objective function value) significantly increases. Therefore, the objective function values obtained under nominal data (i.e., column 4) are larger 
Table 4. Computational results of solving deterministic and robust models under realizations.

\begin{tabular}{|c|c|c|c|c|}
\hline \multirow{2}{*}{$\begin{array}{l}\text { Uncertainty } \\
\text { level }\end{array}$} & \multicolumn{2}{|c|}{$\begin{array}{c}\text { Objective function value } \\
\text { of test problem } 1\end{array}$} & \multicolumn{2}{|c|}{$\begin{array}{c}\text { Objective function value } \\
\text { of test problem } 2\end{array}$} \\
\hline & $\begin{array}{c}\text { Deterministic } \\
\text { model }\end{array}$ & $\begin{array}{c}\text { Robust } \\
\text { model }\end{array}$ & $\begin{array}{c}\text { Deterministic } \\
\text { model }\end{array}$ & $\begin{array}{c}\text { Robust } \\
\text { model }\end{array}$ \\
\hline \multirow{5}{*}{0.25} & 2073941.8 & 2095163.0 & 3397128.0 & 3407425.5 \\
\hline & 2068080.8 & 2099367.6 & 3377570.2 & 3400200.4 \\
\hline & 2075620.7 & 2097972.3 & 3382655.1 & 3405412.3 \\
\hline & 2065084.6 & 2097846.2 & 3400052.8 & 3416850.5 \\
\hline & 2061580.3 & 2094261.2 & 3406637.0 & 3414246.7 \\
\hline \multirow{5}{*}{0.5} & 2076900.1 & 2092975.6 & 3420669.6 & 3569850.7 \\
\hline & 2067783.7 & 2094582.5 & 3405743.9 & 3565026.9 \\
\hline & 2067095.7 & 2093771.0 & 3417193.4 & 3564497.6 \\
\hline & 2066733.7 & 2094779.4 & 3427752.4 & 3576307.1 \\
\hline & 2062146.7 & 2089279.0 & 3413285.6 & 3565070.5 \\
\hline \multirow{5}{*}{0.75} & 2100149.6 & 2107830.0 & 3427752.4 & 3580027.7 \\
\hline & 2062487.0 & 2090989.2 & 3451445.0 & 3553243.1 \\
\hline & 2078472.1 & 2098781.2 & 3430072.4 & 3560741.5 \\
\hline & 2068460.3 & 2097287.2 & 3473769.4 & 3553660.9 \\
\hline & 2079271.0 & 2104729.0 & 3504365.3 & 3569236.5 \\
\hline \multirow{5}{*}{1} & 2110251.8 & 2107153.4 & 3536774.0 & 3654595.0 \\
\hline & 2055914.5 & 2094387.1 & 3526927.1 & 3660368.6 \\
\hline & 2094812.8 & 2107041.2 & 3462342.7 & 3659533.4 \\
\hline & 2078110.2 & 2102250.6 & 3465606.4 & 3654270.8 \\
\hline & 2051609.2 & 2087027.0 & 3536719.0 & 3654104.6 \\
\hline
\end{tabular}

than those obtained under realizations (i.e., column $6)$.

The computational times are reported in the third and fourth columns in Table 3. They show the computational time in seconds for solving the deterministic and robust models. According to these results, it can be affirmed that both deterministic and robust models are solved in a reasonable time.

\subsection{Sensitivity analysis}

After validating the proposed robust model, we conducted a sensitivity analysis to show the usefulness of incorporating the reliability concepts into the proposed model to mitigate the impacts of disruptions. We study the impact of the size of capacity disruptions (i.e., by changing the corresponding capacity failure fraction at unreliable HDC facilities) on the location of reliable and unreliable HDC facilities and their numbers, total network costs, transportation costs, costs of sharing strategy, and the amount of products trans-shipped from reliable HDC facilities to unreliable ones after disruption. To do so, the capacity failure fractions (i.e., $p_{j}=p_{j}^{\prime}$ ) are equally varied. It should be mentioned

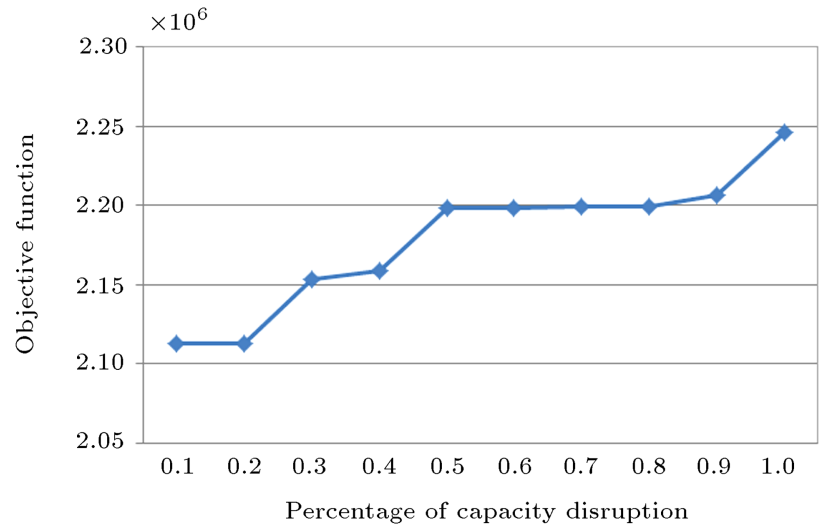

Figure 2. Capacity disruptions vs objective function.

that the sensitivity analysis is carried out on test problem 1 with uncertainty level of 0.25 . The details of the results are reported in Table 5 and graphically depicted in Figures 2-4.

The first column of Table 5 presents the percentage of capacity disrupted at unreliable HDC facilities. The second and the fifth columns illustrate the objective function value and the fixed opening 
Table 5. Results of sensitivity analysis.

\begin{tabular}{cccccccc}
\hline $\boldsymbol{p}_{\boldsymbol{j}}=\boldsymbol{p}_{\boldsymbol{j}}^{\prime}$ & $\begin{array}{c}\text { Objective } \\
\text { function }\end{array}$ & $\boldsymbol{Y} \boldsymbol{R}(\boldsymbol{j})=\mathbf{1}$ & $\boldsymbol{Y} \boldsymbol{U}(\boldsymbol{j})=\mathbf{1}$ & $\begin{array}{c}\text { Opening } \\
\text { costs }\end{array}$ & $\begin{array}{c}\text { Transportation } \\
\text { costs }\end{array}$ & $\begin{array}{c}\text { Disruption } \\
\text { costs }\end{array}$ & $\begin{array}{c}\text { Amount of } \\
\text { shipped } \\
\text { products }\end{array}$ \\
\hline 0.1 & 2112723.6 & 2 & 345 & 2071621 & 41042.6 & 747.0 & 124.5 \\
0.2 & 2112723.6 & 2 & 345 & 2071621 & 41042.6 & 747.0 & 124.5 \\
0.3 & 2153180.2 & 23 & 45 & 2112164 & 40991.7 & 414.8 & 48.5 \\
0.4 & 2158605.7 & 25 & 34 & 2116685 & 41828.3 & 1542.2 & 219.7 \\
0.5 & 2198695.8 & 235 & 4 & 2157228 & 41437.6 & 999.5 & 126.6 \\
0.6 & 2198712.3 & 235 & 4 & 2157228 & 41441.9 & 1407.2 & 175.1 \\
0.7 & 2199148.9 & 235 & 4 & 2157228 & 41865.3 & 1843.4 & 223.6 \\
0.8 & 2199469.7 & 235 & 4 & 2157228 & 42173.1 & 2279.7 & 272.1 \\
0.9 & 2206403.5 & 245 & 3 & 2164957 & 41227.2 & 2738.8 & 335.3 \\
\hline & 2246208.5 & 2345 & - & 2205500 & 40708.2 & 0 & 0 \\
\hline
\end{tabular}

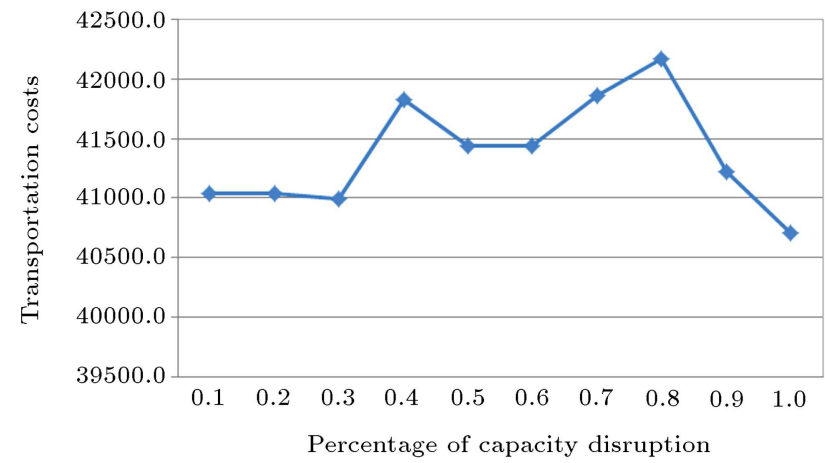

Figure 3. Capacity disruptions vs transportation costs.

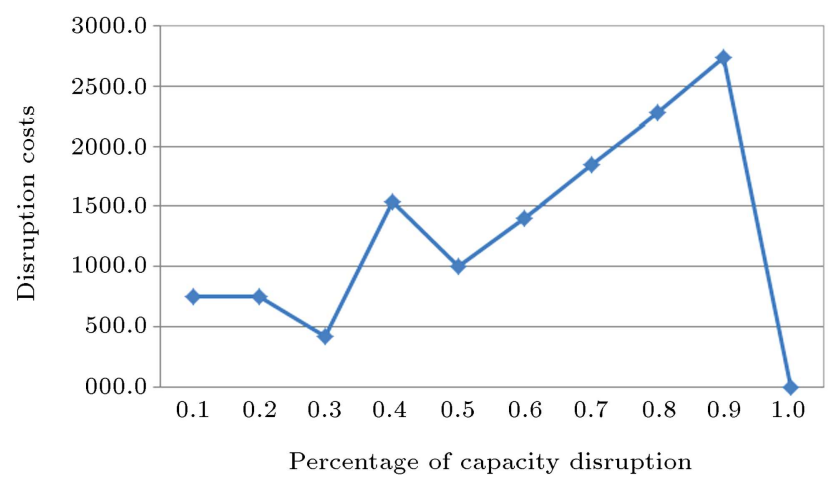

Figure 4. Capacity disruptions vs disruption costs.

costs, respectively. The third and fourth columns show the location of the reliable and unreliable HDC facilities and their opened numbers. The sixth column indicates the transportation costs between facilities and customers. The seventh column reports the disruption $\operatorname{costs}\left(\sum_{j^{\prime}} \sum_{j \neq j^{\prime}} \tilde{d}_{j^{\prime} j} T_{j^{\prime} j}\right)$ associated with the sharing strategy, which are the costs of trans-shipping products from reliable $\mathrm{HDC}$ facilities to unreliable ones in a disruption situation. Furthermore, the last column shows the amounts of trans-shipped products between reliable and unreliable $\mathrm{HDC}$ facilities $\left(\sum_{j^{\prime}} \sum_{j \neq j^{\prime}} T_{j^{\prime} j}\right)$ when capacities are disrupted.
According to the results reported in the first two columns of Table 5, the objective function value increases, when the percentage of capacity disruption is increased. This issue is also depicted in Figure 2. Furthermore, by increasing the capacity failure fractions, the model determines that more reliable HDC facilities must be located. However, when the capacity disruptions are small, most of the located HDC facilities are unreliable. This matter illustrates the impact of capacity disruptions on the location of reliable and unreliable HDC facilities as well as their numbers (see columns 3 and 4 of Table 5).

The fixed opening costs are not changed or increased when capacities are increasingly disrupted. In the cases where the location of reliable and unreliable HDC facilities is not changed by increasing the percentage of capacity disruptions (for example, see rows 6-9 in Table 5), the amount of products transshipped from reliable HDC facilities to unreliable ones and the corresponding costs, i.e. disruption costs, are increased. In these cases, the transportation costs are also increased. Figures 3 and 4 depict the behavior of the disruption costs and transportation costs at different levels of capacity disruptions, respectively. The above discussions approve the application of capacity disruptions, sharing strategy, and other reliability concepts in our proposed model.

\section{Concluding remarks}

This paper offers a robust and reliable model to protect an integrated forward-reverse logistics network against random facility disruptions and, at the same time, to cope with existing uncertainties in the model parameters. To capture random facility disruptions, several reliability strategies and assumptions are taken into account. Random disruption at hybrid HDC facilities is taken into consideration. In this manner, two types of facilities, reliable or unreliable HDC 
facilities, are allowed to be located in the concerned network. Furthermore, partial and complete capacity disruptions and a sharing strategy are also considered, which can improve the service level after occurrence of disruptions. To deal with the uncertainty in the parameters of the network, a robust optimization approach is applied to the original deterministic model. The effect of capacity disruptions on the objective function, opening and transportation costs, disruption costs, and the amount of shared products between HDC facilities are also investigated through a sensitivity analysis.

Finally, some directions are stated for future research. It is possible to incorporate the reliability concepts into the transportation and inventory decisions to design a more reliable supply chain network. Modeling the different types of disruption (caused by natural, man-made, or technological threats) and their impacts on facilities and/or transportation links through a scenario-based approach would be of particular interest.

\section{Acknowledgments}

The authors are grateful to the respected reviewers for their constructive and valuable comments in preparation of the revised manuscript.

\section{References}

1. Fleischmann, M., Beullens, P., Bloemhof-Ruwaard, J.M. and Wassenhove, L. "The impact of product recovery on logistics network design", Production and Operations Management, 10, pp. 156-173 (2001).

2. Lee, D. and Dong, M. "A heuristic approach to logistics network design for end-of-lease computer products recovery", Transportation Research Part E, 44, pp. 455-474 (2007).

3. Lin, L., Gen, M. and Wang, X. "Integrated multistage logistics network design by using hybrid evolutionary algorithm", Computers \& Industrial Engineering, 56, pp. 854-873 (2009).

4. Pishvaee, M.S., Jolai, F. and Razmi, J. "A stochastic optimization model for integrated forward/reverse logistics network design", Journal of Manufacturing Systems, 28, pp. 107-114 (2009).

5. Fleischmann, M., Krikke, H.R., Dekker, R. and Flapper, S.D.P. "A characterisation of logistics networks for product recovery", Omega, 28, pp. 653-666 (2000).

6. Krikke, H., Bloemhof-Ruwaard, J. and Van Wassenhove, L.N. "Concurrent product and closed-loop supply chain design with an application to refrigerators", International Journal of Production Research, 41, pp. 3689-3719 (2003).

7. Kleindorfer, P. and Saad, G. "Managing disruption risks in supply chains", Production and Operations Management, 14, pp. 53-68 (2005).
8. Peng, P., Snyder, L.V., Lim, A. and Liu, Z. "Reliable logistics networks design with facility disruptions", Transportation Research Part B, 45, pp. 1190-1211 (2011).

9. Klibi, W., Martel, A. and Guitouni, A. "The design of robust value-creating supply chain networks: a critical review", European Journal of Operational Research, 203, pp. 283-293 (2010).

10. Listes, O. and Dekker, R. "A stochastic approach to a case study for product recovery network design", European Journal of Operational Research, 160, pp. 268-287 (2005).

11. Salema, M.I.G., Barbosa-Povoa, A.P. and Novais, A.Q. "An optimization model for the design of a capacitated multi-product reverse logistics network with uncertainty", European Journal of Operational Research, 179, pp. 1063-1077 (2007).

12. Listes, O. "A generic stochastic model for supply-andreturn network design", Computers and Operations Research, 34, pp. 417-442 (2008).

13. Ding, H., Benyoucef, L. and Xie, X. "Stochastic multiobjective production-distribution network design using simulation based optimization", International Journal of Production Research, 47, pp. 479-505 (2009).

14. Lee, D. and Dong, M. "Dynamic network design for reverse logistics operations under uncertainty", Transportation Research Part E, 45, pp. 61-71 (2009).

15. El-Sayed, M., Afia, N. and El-Kharbotly, A. "A stochastic model for forward-reverse logistics network design under risk", Computers \& Industrial Engineering, 58, pp. 423-431 (2010).

16. Pishvaee, M.S., Rabbani, M. and Torabi, S.A. "A robust optimization approach to closed-loop supply chain network design under uncertainty", Applied Mathematical Modeling, 35, pp. 637-649 (2011).

17. Hasani, A., Zegordi, S.H. and Nikbakhsh, E. "Robust closed-loop supply chain network design for perishable goods in agile manufacturing under uncertainty", International Journal of Production Research, 50, pp. 4649-4669 (2012).

18. Mula, J., Poler, R. and Garcia, J.P. "MRP with flexible constraints: a fuzzy mathematical programming approach", Fuzzy Sets and Systems, 157, pp. 74-97 (2006).

19. Torabi, S.A. and Hassini, E. "An interactive possibilistic programming approach for multiple objective supply chain master planning", Fuzzy Sets and Systems, 159, pp. 193-214 (2008).

20. Mitra, K., Gudi, R.D., Patwardhan, S.C. and Sardara, G. "Towards resilient supply chains: Uncertainty analysis using fuzzy mathematical programming", Chemical Engineering Research and Design, 87, pp. 967-981 (2009).

21. Pishvaee, M.S. and Torabi, S.A. "A possibilistic programming approach for closed-loop supply chain network design under uncertainty", Fuzzy Sets and Systems, 161, pp. 2668-2683 (2010). 
22. Bouzembrak, Y., Allaoui, H., Goncalves, G., Bouchriha, H. and Baklouti, M. "A possibilistic linear programming model for supply chain network design under uncertainty", IMA Journal of Management Mathematics, 24, pp. 209-229 (2013).

23. Vahdani, B., Razmi, J. and Tavakkoli-Moghaddam, R. "Fuzzy possibilistic modeling for closed loop recycling collection networks", Environmental Modeling and Assessment, 17, pp. 623-637 (2012).

24. Drezner, Z. "Heuristic solution methods for two location problems with unreliable facilities", The Journal of the Operational Research Society, 38, pp. 509-514 (1987).

25. Snyder, L.V. and Daskin, M. "Reliability models for facility location: the expected failure cost case", Transportation Science, 39, pp. 400-416 (2005).

26. Snyder, L.V., Scaparra, M., Daskin, M. and Church, R. "Planning for disruptions in supply chain networks", In TutORials in Operations Research, INFORMS, Greenberg, H., Ed., Baltimore, pp. 234-257 (2006).

27. Berman, O., Krass, D. and Menezes, M. "Facility reliability issues in network p-median problems: strategic centralization and co-location effects", Operations Research, 55, pp. 332-350 (2007).

28. Cui, T., Ouyang, Y. and Shen, Z.J.M. "Reliable facility location design under the risk of disruptions", Operations Research, 58, pp. 998-1011 (2010).

29. Li, X. and Ouyang, Y. "A continuum approximation approach to reliable facility location design under correlated probabilistic disruptions", Transportation Research Part B, 44, pp. 535-548 (2010).

30. Qi, L., Shen, Z.-J.M. and Snyder, L.V. "The effect of supply disruptions on supply chain design decisions", Transportation Science, 44, pp. 274-289 (2010).

31. Lim, M., Daskin, M.S., Bassamboo, A. and Chopra, S. "A facility reliability problem: formulation, properties and algorithm", Naval Research Logistics, 57, pp. 5870 (2010).

32. Azad, N., Saharidis, G.K.D., Davoudpour, H., Malekly, H. and Yektamaram, S.A. "Strategies for protecting supply chain networks against facility and transportation disruptions: an improved Benders decomposition approach", Annals of Operations Research, 210, pp. 125-163 (2012).

33. Davarzani, H., Zegordi, S.H. and Norrman, A. "Contingent management of supply chain disruption: Effects of dual or triple sourcing", Scientia Iranica, Transactions E: Industrial Engineering, 18, pp. 15171528 (2011).

34. Lim, M.K., Bassamboo, A., Chopra, S. and Daskin, M.S. "Facility location decisions with random disruptions and imperfect estimation", Manufacturing and Service Operations Management, 15, pp. 239-249 (2013).

35. Aboolian, R., Cui, T. and Shen, Z.J.M. "An efficient approach for solving reliable facility location models", INFORMS Journal on Computing, 25, pp. 720-729 (2013).
36. Baghalian, A., Rezapour, S. and Zanjirani Farahani, R. "Robust supply chain network design with service level against disruptions and demand uncertainties: A reallife case", European Journal of Operational Research, 227, pp. 199-215 (2013).

37. Babazadeh, R. and Razmi, J. "A robust stochastic programming approach for agile and responsive logistics under operational and disruption risks", International Journal of Logistics Systems and Management, 13, pp. 458-482 (2012).

38. Garcia-Herreros, P., Grossmann, I.E. and Wassick, J. "Design of supply chains under the risk of facility disruptions", Computer Aided Chemical Engineering, 32, pp. 577-582 (2013).

39. Vahdani, B., Tavakkoli-Moghaddam, R. and Jolai, F. "Reliable design of a logistics network under uncertainty: A fuzzy possibilistic-queuing model", Applied Mathematical Modelling, 37, pp. 3254-3268 (2013).

40. Vahdani, B., Tavakkoli-Moghaddam, R., Jolai, F. and Baboli, A. "Reliable design of a closed loop supply chain network under uncertainty: An interval fuzzy possibilistic chance constrained model", Engineering Optimization, 45, pp. 745-765 (2013).

41. Vahdani, B., Tavakkoli-Moghaddam, R., Modarres, M. and Baboli, A. "Reliable design of a forward/reverse logistics network under uncertainty: A robust-M/M/c queuing model", Transportation Research Part E: Logistics and Transportation Review, 48, pp. 1152-1168 (2012).

42. Hatefi, S.M. and Jolai, F. "Robust and reliable forward-reverse logistics network design under demand uncertainty and facility", Applied Mathematical Modelling, 38, pp. 2630-2647 (2014).

43. Hatefi, S.M. and Jolai, F. "Reliable forward-reverse logistics network design under partial and complete facility disruptions", International Journal of Logistics Systems and Management, 20, pp. 370-394 (2015).

44. Bertsimas, D. and Sim, M. "Robust discrete optimization and network flows", Mathematical Programming Series B., 98, pp. 49-71 (2003).

45. Bertsimas, D. and Sim, M. "The price of robustness", Operations Research, 52, pp. 35-53 (2004).

46. Easwaran, G. and Üster, H. "A closed-loop supply chain network design problem with integrated forward and reverse channel decisions", IIE Transactions, 42, pp. 779-792 (2010).

47. Soyster, A.L. "Convex programming with set-inclusive constraints and applications to inexact linear programming", Operations Research, 21, pp. 1154-1157 (1973).

48. El-Ghaoui, L. and Lebert, H. "Robust solutions to least-square problems to uncertain data matrices", SIAM Journal on Matrix Analysis and Applications, 18, pp. 1035-1064 (1997).

49. El-Ghaoui, L., Oustry, F. and Lebert, H. "Robust solutions to uncertain semi-definite programs", SIAM Journal on Optimization, 9, pp. 33-52 (1998). 
50. Ben-Tal, A. and Nemirovski, A. "Robust convex optimization", Mathematics of Operations Research, 2, pp. 769-805 (1998).

51. Ben-Tal, A., Nemirovski, A. "Robust solutions to uncertain linear programs", Operations Research Letters, 25, pp. 1-13 (1999).

52. Ben-Tal, A. and Nemirovski, A. "Robust solutions of linear programming problems contaminated with uncertain data", Mathematical Programming, 88, pp. 411-424 (2000).

53. Ben-Tal, A., Golany, B., Nemirovski, A. and Vial, J.P. "Retailer-supplier flexible commitments contracts: a robust optimization approach", Manufacturing and Service Operations Management, 7, pp. 248-271 (2005).

54. Ben-Tal, A., El-Ghaoui, L. and Nemirovski, A., Robust Optimization, Princeton University Press (2009).

55. Melo, M.T., Nickel, S. and Saldanha da Gama F. "Facility location and supply chain management - A review", European Journal of Operational Research, 196, pp. 401-412 (2009).

56. Meepetchdee, Y. and Shah, N. "Logistical network design with robustness and complexity considerations", Int. J. Phys. Distrib. Log. Manage., 37, pp. 201-222 (2007).

57. Pishvaee, M.S., Razmi, J. and Torabi, S.A. "Robust possibilistic programming for socially responsible supply chain network design: A new approach", Fuzzy Sets and Systems, 206, pp. 1-20 (2012).

\section{Biographies}

Seyed Morteza Hatefi is currently an Assistant Professor in the Faculty of Engineering at Shahrekord University, Iran. He received his BS degree in Statistics from Shahid Beheshti University, Tehran, in 2006, and his $\mathrm{MS}$ and $\mathrm{PhD}$ degrees in Industrial Engineering from University of Tehran, Tehran, in 2009 and 2013, respectively. His current research interests include: supply chain network design, logistics systems, multicriteria decision making, data envelopment analysis, performance measurement and management, operations research applications, construction and project management.

Fariborz Jolai is currently professor of Industrial Engineering at College of Engineering, University of Tehran, Tehran, Iran. He obtained his PhD degree in Industrial Engineering from INPG, Grenoble, France, in 1998. His current research interests are scheduling and production planning, supply chain modeling, and optimization problems under uncertainty conditions.

Seyed Ali Torabi is currently Associate Professor of Industrial Engineering at College of Engineering, University of Tehran, Tehran, Iran. His current research interests are supply chain modeling, disaster management, multi-criteria decision making, fuzzy mathematical programing, inventory management, and optimization problems under uncertainty conditions.

Reza Tavakkoli-Moghaddam is currently professor of Industrial Engineering at College of Engineering, University of Tehran, Tehran, Iran. He received his MS degree in Industrial Engineering from University of Melbourne and PhD degree from Swinburne University of Technology. He is Professor of Industrial Engineering at University of Tehran in Iran. He consulted for a number of manufacturing and service firms. His research is directed towards facilities layout and location design, cellular manufacturing system, sequencing and scheduling, and using meta-heuristics for combinatorial optimization problems. He is the author of over 100 journal papers and 150 papers in conference proceedings. 